\title{
Factorizations for Self-Dual Gauge Fields ${ }^{\star}$
}

\author{
David E. Lerner \\ Department of Mathematics, University of Kansas, Lawrence, KS 66045, USA
}

\begin{abstract}
For a particular class of patching matrices on $P_{3}(\mathbb{C})$, including those for the complex instanton bundles with structure group $\operatorname{Sp}(k, \mathbb{C})$ or $O(2 k, \mathbb{C})$, we show that the associated Riemann-Hilbert problem $G(x, \lambda)=$ $G_{-}(x, \lambda) \cdot G_{+}^{-1}(x, \lambda)$ can be generically solved in the factored form $G_{-}=$ $\phi_{1} \cdot \phi_{2} \cdots \cdot \phi_{n}$. If $\Gamma=\Gamma_{n}$ is the potential generated in the usual way from $G_{-}$, and we set $\psi_{i}=\phi_{1} \cdot \cdots \cdot \phi_{i}$, with $\psi_{n}=G_{-}$, then each $\psi_{i}$ also generates a selfdual gauge potential $\Gamma_{i}$. The potentials are connected via the "dressing transformations"

$$
\Gamma_{i}=\phi_{i}^{-1} \cdot \Gamma_{i-1} \cdot \phi_{i}+\phi_{i}^{-1} D \phi_{i}
$$

of Zakharov-Shabat. The factorization is not unique; it depends on the (arbitrary) ordering of the poles of the patching matrix.
\end{abstract}

\section{Introduction}

In general, it is difficult to solve the Riemann-Hilbert problem associated with Ward's construction of self-dual gauge fields [Wa]. Some time ago, Atiyah and Ward wrote down an upper triangular ansatz for the rank-2 instanton bundles [AW]; this problem was then solved explicitly by Corrigan, et. al. in [CFGY]. For bundles of higher rank, algebraic methods do not (to the author's knowledge) yield upper triangular matrices. Nevertheless, as we show below, for the groups $\operatorname{Sp}(k, \mathbb{C})$ and $O(2 k, \mathbb{C})$, patching matrices can be found that allow the RiemannHilbert problem to be solved generically in a finite number of steps by means of residues or partial fractions.

To state the main result, let $G: P_{3}(\mathbb{C}) \rightarrow \operatorname{Sp}(k, \mathbb{C})$ be a rational map given in homogeneous coordinates by $G(Z)=\Delta_{-}^{-1}(Z) \cdot \Delta_{+}^{-1}(Z) \cdot S(Z)$, where $\Delta_{-}, \Delta_{+}$are relatively prime homogeneous polynomials of degree $n$, and $S$ is a matrix of homogeneous polynomials of degree $2 n$. Let $V_{ \pm}=\left\{Z: \Delta_{ \pm}(Z)=0\right\}$ and $U_{ \pm}=P_{3}(\mathbb{C}) \backslash V_{ \pm}$, and let $\mathscr{P}=U_{+} \cup U_{-}$. Let $\mathscr{M}$ be the open subset of the Grassmannian $\operatorname{Gr}(2,4)$ whose points $x$ correspond to projective lines $L_{x}$ lying in $\mathscr{P}$. The patching matrix $G$ defines a $2 k$-dimensional vector bundle $\mathscr{E}$ on $\mathscr{P}$, and we suppose that for some $x, \mathscr{E} \mid L_{x}$ is trivial. Then we shall show

\footnotetext{
* Supported by the General Research Fund of the University of Kansas
} 
Theorem. For a generic $G$ as above, there exists a Zariski-open subset $\mathscr{U}$ of $\mathscr{M}$ such that for $x \in \mathscr{U}, G \mid L_{x}$ factors as $G_{-}(x, \lambda) \cdot G_{+}^{-1}(x, \lambda)$, with $G_{ \pm}(x, \lambda): U_{ \pm} \cap L_{x} \rightarrow \operatorname{Sp}(k, \mathbb{C})$, and

$$
G_{-}(x, \lambda)=\phi_{1}(x, \lambda) \cdots \cdot \phi_{n}(x, \lambda)
$$

with each $\phi_{i}(x, \lambda)$ of the form $I-A_{i}(x, \lambda)$. $A_{i}$ varies algebraically with $x$, and for each $\lambda$ in its domain, is a nilpotent of order 2 in $\operatorname{sp}(k, \mathbb{C})$. An identical result holds for the groups $O(2 k, \mathbb{C})$.

From the construction, it follows immediately that if $\psi_{1}=\phi_{1}, \psi_{2}=\phi_{1} \cdot \phi_{2}, \ldots, \psi_{n}=$ $\phi_{1} \cdots \cdot \phi_{n}=G_{-}$, the quantities

$$
\Gamma_{j A}(x, \lambda):=\psi_{j}^{-1} \cdot D_{A} \psi_{j}, \quad \text { for } j=1, \ldots, n
$$

all determine self-dual gauge potentials. (The differential operators $D_{A}$ are defined below.) They may be "generated" from $\Gamma_{0 A}=I$ by a sequence of transformations of the form

$$
\Gamma_{j A}=\phi_{j}^{-1} \cdot \Gamma_{j-1, A} \cdot \phi_{j}+\phi_{j}^{-1} \cdot D_{A} \phi_{j}
$$

Although our motivation comes from looking at the original monad or ADHM construction [ADHM], the factorization does not depend on the rationality of $G$. It can be obtained (in general) whenever $G \mid L_{x}$ is meromorphic with a suitable pole structure; in particular, $G$ need not originate with the ADHM construction.

The factorization is not unique; it depends (as does the set $\mathscr{U}$ ) on the (arbitrary) ordering of the $n$ poles of $\Delta_{-} \mid L_{x}$. This is a partial analogue, for self-dual gauge fields, of Uhlenbeck's factorization theorem for harmonic maps [Uh].

In the first section of this paper, we review the ADHM construction and demonstrate the existence of patching matrices having a particular form. Section 2 connects this with Ward's construction and the Riemann-Hilbert problem. In Sect. 3 we show how to factor rational maps into the complex symplectic groups, and use this in Sect. 4 to attack the Riemann-Hilbert problem for self-dual gauge fields. Section 5 briefly mentions some consequences of the preceding results.

\section{Algebraic Charts for the ADHM Construction}

Assume in what follows that $E$ is an algebraic vector bundle of rank $2 k$ on $P_{3}(\mathbb{C})$, trivial over the generic line, arising from the monad construction of Barth and Horrocks. (See [ADHM, At, Do, OSS] and references quoted therein.) We shall suppose the structure group to be $\operatorname{Sp}(k, \mathbb{C})$, the case of $O(2 k, \mathbb{C})$ being essentially identical. Thus $E$ is determined by a map $\mathscr{A}(Z): \mathbb{H} \rightarrow(\mathbb{K}, \Omega)$, where $\mathbb{H}$ and $\mathbb{K}$ are comblex vector spaces of dimension $n$ and $2 n+2 k$ respectively, $\Omega$ is a nondegenerate symplectic form on $\mathbb{K}$, and $Z \in \mathbb{C}^{4} \backslash\{0\}$. The requirements on $\mathscr{A}(Z)$ are (1) that it be injective, (2) that the $\operatorname{map} \mathscr{B}(Z): \mathbb{K} \rightarrow \mathbb{H}^{*}$ defined by $\mathscr{B}(Z)=\mathscr{A}^{t}(Z) \Omega$ be surjective, (3) that $\mathscr{B}(Z) \circ \mathscr{A}(Z)=0,(4)$ that $\mathscr{A}(Z)$ be linear in $Z$ and finally (5) that there exist a pair $(X, Y)$ such that $\mathscr{B}(Y) \circ \mathscr{A}(X)$ is an isomorphism. The reality conditions [ADHM] guaranteeing that $E \mid L_{x}$ is trivial for $x \in S^{4} \subset \operatorname{Gr}(2,4)$ are not important in what follows. We choose and fix bases in $\mathbb{H}$ and $\mathbb{K}$, and take $\Omega$ in 
the specific form

$$
\Omega=\left[\begin{array}{cc}
\Omega_{n} & 0 \\
0 & \Omega_{k}
\end{array}\right], \text { where } \Omega_{m}=\left[\begin{array}{cc}
0 & -I_{m} \\
I_{m} & 0
\end{array}\right] .
$$

The bundle $E$ is defined as $\operatorname{Ker} \mathscr{B} / \operatorname{Im} \mathscr{A}$, and we seek charts on $P_{3}(\mathbb{C})$ over which $E$ is algebraically trivial. Specifically, we require charts on $P_{3}(\mathbb{C})$ of the form $U_{a}=P_{3}(\mathbb{C}) \backslash V_{a}$, where $V_{a}$ is the zero set of a homogeneous polynomial $\Delta_{a}(Z)$. In addition, we want $\operatorname{Ker} \mathscr{B} \mid U_{a}$ to decompose as the direct $\operatorname{sum}\left(\operatorname{Im} \mathscr{A} \mid U_{a}\right) \oplus F_{a}$, where the decomposition is algebraic - i.e., given by rational maps. The patching matrices defined on $U_{a} \cap U_{b}$ for $\operatorname{Ker} \mathscr{B} \rightarrow P_{3}(\mathbb{C})$, will then be block upper triangular, with the lower right-hand blocks $G_{a b}(Z)$ giving the patching for $E$ over $U_{a} \cup U_{b}$. Such charts are readily obtained:

For each $Z$, the $n \times(2 n+2 k)$ matrix $\mathscr{B}(Z)$ has rank $n$, so it contains at least one $n \times n$ invertible submatrix. Running through the different possibilities will give $\left(\begin{array}{c}2 n+2 k \\ n\end{array}\right)$ charts for $\operatorname{Ker} \mathscr{B}$. In particular, let $a=\left(i_{1}, \ldots, i_{n}\right)$ be an $n$-tuple with $1 \leqq i_{1} \leqq \cdots \leqq i_{n} \leqq 2 n+2 k$, let $b_{j}(Z)$ be the $j^{\text {th }}$ column of $\mathscr{B}(Z)$, and let $P_{a}$ be a nonsingular matrix such that $\mathscr{B}_{a}(Z):=\mathscr{B}(Z) P_{a}=\left(b_{i_{1}}|\cdots| b_{i_{n}} \mid *\right)$. Let $\Delta_{a}(Z)=\operatorname{Det}\left(b_{i_{1}} \mid \cdots\right.$ $\left.\mid b_{i_{n}}\right)(Z)$; this is a homogeneous polynomial of degree $n$. Let $V_{a}=\left\{Z: \Delta_{a}(Z)=0\right\}$, and let $U_{a}$ be the complement of $V_{a}$ in $P_{3}(\mathbb{C})$. By the assumptions on $\mathscr{A}(Z)$, the collection $\left\{U_{a}\right\}$ is a Zariski open cover of $P_{3}(\mathbb{C})$.

If $Y$ lies in $\operatorname{Ker} \mathscr{B} \mid U_{a}$, we write

$$
P_{a}^{-1} Y=\left[\begin{array}{c}
\xi_{a} \\
\eta_{a}
\end{array}\right], \quad \text { and } \quad \mathscr{B}_{a}(Z)=\left[\alpha_{a}(Z) \mid \beta_{a}(Z)\right] \text {, }
$$

where $\alpha_{a}$ is $n \times n$ and $\xi_{a}$ is $n \times 1$. Since $\alpha_{a}$ is invertible, $\mathscr{B}(Z) Y=0=\mathscr{B}_{a}(Z) P_{a}^{-1} Y$ gives $\xi_{a}=-\alpha_{a}^{-1}(Z) \beta_{a}(Z) \eta_{a}$, and we can define a chart $\Psi_{a}: U_{a} \times \mathbb{C}^{n+2 k} \rightarrow \operatorname{Ker} \mathscr{B} \mid U_{a}$ by

$$
\Psi_{a}\left(Z, \eta_{a}\right)=P_{a}\left[\begin{array}{c}
-\alpha_{a}^{-1}(Z) \beta_{a}(Z) \\
I_{n+2 k}
\end{array}\right] \cdot \eta_{a} .
$$

If $Z \in U_{a} \cap U_{b}$, then for some $\eta_{a}$ and $\eta_{b}$,

$$
Y=P_{a}\left[\begin{array}{c}
-\alpha_{a}^{-1}(Z) \beta_{a}(Z) \\
I_{n+2 k}
\end{array}\right] \cdot \eta_{a}=P_{b}\left[\begin{array}{c}
-\alpha_{b}^{-1}(Z) \beta_{b}(Z) \\
I_{n+2 k}
\end{array}\right] \cdot \eta_{b},
$$

and it follows that $\eta_{a}=K_{a b}(Z) \eta_{b}$, where

$$
K_{a b}(Z)=\tau \circ P_{a}^{-1} \circ P_{b}\left[\begin{array}{c}
-\alpha_{b}^{-1}(Z) \beta_{b}(Z) \\
I_{n+2 k}
\end{array}\right],
$$

$\tau$ being the projection onto the last $n+2 k$ components. Notice that $K_{a b}$ is $\Delta_{b}^{-1}(Z)$ times a matrix of homogeneous polynomials of degree $n$.

Let $A_{j}(Z)$ be the $j^{\text {th }}$ column of $\mathscr{A}(Z)$, so that $\operatorname{Im} \mathscr{A}(Z)=\operatorname{span}\left\{A_{j}(Z): 1 \leqq j \leqq n\right\}$. The $\left\{A_{j}(Z)\right\}$ are linearly independent in $\mathbb{K}$ for all $Z \neq 0$, and since $\operatorname{Im} \mathscr{A} \subset \operatorname{Ker} \mathscr{B}$, the matrices $A^{a}(Z):=\tau \circ P_{a}^{-1} \mathscr{A}(Z)$ are of rank $n$ in $U_{a}$. We are looking for algebraic 
complements to $\operatorname{Im} \mathscr{A}$ in $\operatorname{Ker} \mathscr{B}$; it will be convenient to isolate a subcollection of the $\left\{U_{a}\right\}$ on which these can be found without further refinement of the charts.

Proposition 1. For $2^{n}(1+n k)$ of the charts described above, $P_{a}$ may be chosen so that

(a) $P_{a} \in \mathrm{Sp}(k+n, \mathbb{C})$.

(b) The top $n \times n$ block of $A^{a}(Z)$ is $-\alpha_{a}^{t}(Z)$.

In particular, the matrix

$$
R_{a}(Z):=\left[A^{a}(Z) \mid \begin{array}{l}
0_{n \times 2 k} \\
I_{2 k}
\end{array}\right]
$$

is invertible in $U_{a}$.

Proof. It is readily checked that the following substitutions in $\mathscr{B}(Z)$ are effected by matrices satisfying the above conditions:

1. For $1 \leqq j \leqq n,\{-\operatorname{col}(j) \rightarrow \operatorname{col}(n+j), \operatorname{col}(n+j) \rightarrow \operatorname{col}(j)\}$,

2. For $1 \leqq j \leqq n$, and $1 \leqq m \leqq k,\{\operatorname{col}(j) \leftrightarrow \operatorname{col}(2 n+m), \operatorname{col}(n+j) \leftrightarrow \operatorname{col}(2 n+k+m\}$.

We get $2^{n}$ charts from (1). Composing a transformation of type (2) with one of type (1), we can put any of the last $2 k$ columns into any one of the first $n$ slots. There are then $2^{n-1}$ possible replacements for the remaining $n-1$ slots coming from additional transformations of type (1) for a total of $2^{n}+2 k n \cdot 2^{n-1}=2^{n}(1+n k)$ charts.

The columns of $R_{a}(Z)$ then give the desired direct sum decomposition over $U_{a}$. To trivialize $\operatorname{Ker} \mathscr{B} \mid U_{a}$ and $\operatorname{Ker} \mathscr{B} \mid U_{b}$ using this, we should have to divide the first $n$ columns of $R_{a}(Z)$ and $R_{b}(Z)$ by, say $Z^{\alpha}$ and $Z^{\beta}$ respectively to obtain objects homogeneous of degree 0 ; it turns out that the resulting factor of $Z^{\alpha} / Z^{\beta}$ drops out of the quotient block, so that $E$ is algebraically trivial over $U_{a}$, and we omit this step.

Let $Y, W \in E_{z}$. If $Z \in U_{a}$, the symplectic form on $E$ is defined by $\omega(Y, W)=\Omega\left(\Psi_{a} \cdot Y_{a}, \Psi_{a} \cdot W_{a}\right)$, where $Y_{a}$ and $W_{a}$ are local representatives of the equivalence classes. For the charts described above, we may choose unique local representatives of the form

$$
Y_{a}=R_{a}(Z) \cdot\left[\begin{array}{l}
0_{n} \\
y_{a}
\end{array}\right]=\left[\begin{array}{l}
0_{n} \\
y_{a}
\end{array}\right] .
$$

An easy computation then gives $\omega(Y, W)=y_{a}^{t} \cdot \Omega_{k} \cdot w_{a}$, and if $Z \in U_{b}$ as well, we get $\omega(Y, W)=y_{b}^{t} \cdot \Omega_{k} \cdot w_{b}$. Thus the patching matrix for the quotient given by the lower right-hand block of

$$
R_{a}^{-1} K_{a b} R_{b}=\left[\begin{array}{cc}
* & * \\
0 & G_{a b}
\end{array}\right]
$$

preserves the form $\Omega_{k}$, and we have

Proposition 2. The quotient bundle $E$ is algebraically trivial over each of the charts in Proposition 1. In the intersection of two such charts, the patching matrix takes values in $\operatorname{Sp}(k, \mathbb{C})$ and has the form

$$
G_{a b}(Z)=\Delta_{a}^{-1}(Z) \cdot \Delta_{b}^{-1}(Z) \cdot S_{a b}(Z),
$$


where $\Delta_{a}$ and $\Delta_{b}$ are homogeneous polynomials of degree $n$, and the entries of $S_{a b}$ are homogeneous polynomials of degree $2 n$.

(The last assertion follows on inspection of $R_{a}^{-1} K_{a b} R_{b}$.)

The matrices $G_{a b}$ are not difficult to construct; for example, taking $P_{1}=I$, $P_{2}=\Omega$, if we write

$$
\mathscr{B}(Z)=[\alpha|\rho| \kappa \mid \tau],
$$

where $\alpha, \rho, \kappa$ and $\tau$ have $n, n, k$ and $k$ columns respectively, the lower right block of $R_{1}^{-1} K_{12} R_{2}$ is

$$
G_{12}(Z)=\left[\begin{array}{cc}
-\tau^{t} \alpha^{t^{-1}} \rho^{-1} \tau & \tau^{t} \alpha^{t^{-1}} \rho^{-1} \kappa-I_{k} \\
\kappa^{t} \alpha^{t^{-1}} \rho^{-1} \tau+I_{k} & -\kappa^{t} \alpha^{t^{-1}} \rho^{-1} \kappa
\end{array}\right] .
$$

In what follows, we shall only require one pair $\left(U_{a}, U_{b}\right)$ from the above collection, and we shall take $\Delta_{a}$ and $\Delta_{b}$ to be relatively prime, which holds in the general case.

\section{The Relation to Ward's Construction}

If $x \in \operatorname{Gr}(2,4)$, let $L_{x}$ denote the corresponding line in $P_{3}(\mathbb{C})$. Ward's construction [Wa] begins by restricting both the cover and the patching matrices to projective lines. Using the fact that $E \mid L_{x}$ is trivial for generic lines (a consequence of the assumptions on $\mathscr{A}(Z)$ above), the restricted patching matrices on such lines split as $G_{a b} \mid L_{x}=G_{a}(x, \lambda) \cdot G_{b}^{-1}(x, \lambda)$, with $G_{a}(x, \lambda), G_{b}(x, \lambda)$ holomorphic in $\mathfrak{U}_{a}:=U_{a} \cap L_{x}$, $\mathfrak{U}_{b}:=U_{b} \cap L_{x}$ respectively. Here $\lambda$ is a complex coordinate on $L_{x}$, and $x$ appears parametrically; $G_{a}$ and $G_{b}$ can both be taken to depend holomorphically on $x$. In an affine chart $\cong \mathbb{C}^{4}$ on $\operatorname{Gr}(2,4)$, one can write $x$ as a $2 \times 2$ complex matrix so that $G_{a b}(Z) \mid L_{x}$ takes the form $G_{a b}(x \cdot \pi, \pi)$, where $\pi=\left(\pi_{0}, \pi_{1}\right)$ are homogeneous coordinates on $L_{x}[\mathrm{PR}]$. Defining the linear operators

$$
D_{A}=\pi_{1} \partial / \partial x^{A 0}-\pi_{0} \partial / \partial x^{A 1} \quad(A=0,1),
$$

the functional form of $G_{a b}$ now gives $D_{A} G_{a b}(x \cdot \pi, \pi)=0$, which leads to

$$
G_{a}^{-1}\left(D_{A} G_{a}\right)=G_{b}^{-1}\left(D_{A} G_{b}\right) \quad \text { in } \quad \mathfrak{U}_{a} \cup \mathfrak{U}_{b} .
$$

The global quantity defined on $L_{x}$ by expression (15) is holomorphic and homogeneous of degree 1 in $\pi$; it is thus linear in $\pi$ and can be written as $\Gamma_{A 0}(x) \pi_{1}-\Gamma_{A 1}(x) \pi_{0}$ for $A=0,1$. The potential defined by $\Gamma:=\Gamma_{A B} d x^{A B}$ is then self-dual (or anti self-dual, depending on conventions) by virtue of the fact that $\left[D_{A}, D_{B}\right]=0$. Given the above, we observe that it is only necessary to split one of the $G_{a b}(x \cdot \pi, \pi)$ to obtain $\Gamma$. This is the Riemann-Hilbert problem under discussion.

From now on, we take $G_{a b}$ in the form given by Proposition 2 above. A "generic" point $x$ in the 4-dimensional Grassmannian $\operatorname{Gr}(2,4)$ refers to a line $L_{x} \subset P_{3}(\mathbb{C})$ such that (1) $L_{x} \cap V_{a} \cap V_{b}=\phi$, (2) $L_{x}$ is in general position with respect to $V_{a}$ and $V_{b}$ (so that it intersects each in $n$ distinct points), and (3) $E \mid L_{x}$ is trivial. Thus for generic $L_{x}$, we shall have (1) $L_{x} \subset \mathfrak{U}_{a} \cup \mathfrak{U}_{b}$, (2) $\mathfrak{U}_{a} \cong P_{1}(\mathbb{C}) \backslash\left\{p_{1}(x), \ldots, p_{n}(x)\right\}$, $\mathfrak{U}_{b} \cong P_{1}(\mathbb{C}) \backslash\left\{q_{1}(x), \ldots, q_{n}(x)\right\}$, the deleted points corresponding to the sets $V_{a} \cap L_{x}$ and $V_{b} \cap L_{x}$ respectively, and (3) $\left\{p_{1}(x), \ldots, p_{n}(x)\right\} \cap\left\{q_{1}(x), \ldots, q_{n}(x)\right\}=\phi$. 
Restricted to $L_{x}$, the functions $\Delta_{a}, \Delta_{b}$ and the entries of $S_{a b}$ become homogeneous polynomials in the components of $x$ and $\pi$. Assuming the point corresponding to $\pi=(0,1)$ does not coincide with one of the $p_{i}(x)$, we set $\lambda=\pi_{1} / \pi_{0}, \Lambda=(1, \lambda)$ and conclude that $G(x \cdot \pi, \pi)$ which is homogeneous of degree zero in $\pi$, can be written as

$$
G(x \cdot \Lambda, \Lambda)=G(x, \lambda)=\prod_{1}^{n}\left[\lambda-p_{i}(x)\right]^{-1} \cdot \prod_{1}^{n}\left[\lambda-q_{j}(x)\right]^{-1} \cdot \tilde{S}(x, \lambda),
$$

where we have dropped the indices on the matrices and absorbed a rational function of $x$ into the original $S(x, \lambda)$. Strictly speaking, the $(x, \lambda)$ are local coordinates on the flag manifold $\mathscr{F}_{12}$ consisting of all ordered pairs \{line in $P_{3}(\mathbb{C})$, point on the line) $\}$; see Wells [We]. The Riemann-Hilbert problem is then to find a Zariski-open set $\mathscr{U} \subset \operatorname{Gr}(2,4)$ such that $x \in \mathscr{U} \Rightarrow G(x, \lambda)$ factors as $G_{-}(x, \lambda) \cdot G_{+}^{-1}(x, \lambda)$, with $G_{-}(x, \lambda)$ (respectively $\left.G_{+}(x, \lambda)\right)$ holomorphic in $\hat{\mathfrak{U}}_{a} \mid \mathscr{U}$ (respectively $\left.\hat{\mathfrak{U}}_{b} \mid \mathscr{U}\right)$, where $\hat{\mathfrak{U}}_{i}=\left.\mathscr{F}_{12}\right|_{\mathbb{C}^{4}} \backslash\left\{(x, \pi): \Delta_{i}(x \cdot \pi, \pi)=0\right\}$.

\section{Factoring Maps into $\operatorname{Sp}(k, \mathbb{C})$}

Suppose $D$ is a closed disk centered at $p$ in the complex $\lambda$ plane and that $G: D \backslash\{p\} \rightarrow \operatorname{Sp}(k, \mathbb{C})$ is holomorphic with a simple pole at $\lambda=p$. Then $G(\lambda)=$ $(\lambda-p)^{-1} G_{-1}+G_{0}+(\lambda-p) H(\lambda)$, with $H$ holomorphic in $D$. Write $G_{-1}$ and $G_{0}$ in block form:

$$
G_{m}=\left[\begin{array}{ll}
\alpha_{m} & \beta_{m} \\
\gamma_{m} & \delta_{m}
\end{array}\right], \quad \text { where the entries are } k \times k \text { blocks, }
$$

and define $\chi=\gamma_{-1}^{t} \alpha_{0}-\alpha_{-1}^{t} \gamma_{0}$, and $\hat{\chi}=\delta_{-1}^{t} \beta_{0}-\beta_{-1}^{t} \delta_{0}$. Finally, suppose that $\chi$, $\alpha_{-1}$, and $\delta_{-1}$ are invertible. Then we have

Lemma 3. Under the assumptions stated,

(a) The following expressions for the $2 k \times 2 k$ matrix $A$ are identical:

$$
A=\left[\begin{array}{ll}
-\alpha_{-1} \chi^{-1} \gamma_{-1}^{t} & \alpha_{-1} \chi^{-1} \alpha_{-1}^{t} \\
-\gamma_{-1} \chi^{-1} \gamma_{-1}^{t} & \gamma_{-1} \chi^{-1} \alpha_{-1}^{t}
\end{array}\right]=\left[\begin{array}{ll}
-\beta_{-1} \hat{\chi}^{-1} \delta_{-1}^{t} & \beta_{-1} \hat{\chi}^{-1} \beta_{-1}^{t} \\
-\delta_{-1} \hat{\chi}^{-1} \delta_{-1}^{t} & \delta_{-1} \hat{\chi}^{-1} \beta_{-1}^{t}
\end{array}\right] .
$$

(b) $A \in \operatorname{sp}(k, \mathbb{C}) ; A^{2}=0 ; I+(\lambda-p)^{-1} A=\exp \left[(\lambda-p)^{-1} A\right] \in \operatorname{Sp}(k, \mathbb{C})$ for $\lambda \neq p$.

(c) $\left[I+(\lambda-p)^{-1} A\right] \cdot G(\lambda)$ is a holomorphic map from $D$ to $\operatorname{Sp}(k, \mathbb{C})$.

Proof. Writing out the left-hand side of (c), we see that $A$ must satisfy the (apparently) overdetermined system of equations

$$
A G_{-1}=0, \quad A G_{0}+G_{-1}=0 .
$$

The system turns out to be consistent provided that (a) holds; as shown below, this is a consequence of the identities on the Laurent coefficients resulting from the requirement that $G(\lambda) \in \operatorname{Sp}(k, \mathbb{C})$. The inverses of $\alpha_{-1}$ and $\delta_{-1}$ are required here. Once (a) is established, (c) follows directly. Condition (b) is immediate from the identities below and the form of $A$. We remark that invertibility of the matrices 
required is generic. To verify (a) write

$$
G(\lambda)=\left[\begin{array}{ll}
\alpha(\lambda) & \beta(\lambda) \\
\gamma(\lambda) & \delta(\lambda)
\end{array}\right]
$$

Then $G^{t}(\lambda) \Omega_{k} G(\lambda)=\Omega_{k}$ is equivalent to

$$
\gamma^{t} \alpha=\alpha^{t} \gamma, \quad \delta^{t} \beta=\beta^{t} \delta, \quad \alpha^{t} \delta-\gamma^{t} \beta=I_{k},
$$

which gives conditions on the components of $G_{m}$ :

$$
\begin{aligned}
& \gamma_{-1}^{t} \alpha_{-1}=\alpha_{-1}^{t} \gamma_{-1}, \quad \gamma_{-1}^{t} \alpha_{0}+\gamma_{0}^{t} \alpha_{-1}=\alpha_{-1}^{t} \gamma_{0}+\alpha_{0}^{t} \gamma_{-1}, \\
& \delta_{-1}^{t} \beta_{-1}=\beta_{-1}^{t} \delta_{-1}, \quad \delta_{-1}^{t} \beta_{0}+\delta_{0}^{t} \beta_{-1}=\beta_{-1}^{t} \delta_{0}+\beta_{0}^{t} \delta_{-1} \text {, } \\
& \alpha_{-1}^{t} \delta_{-1}=\gamma_{-1}^{t} \beta_{-1}, \quad \alpha_{-1}^{t} \delta_{0}+\alpha_{0}^{t} \delta_{-1}=\gamma_{-1}^{t} \beta_{0}+\gamma_{0}^{t} \beta_{-1} .
\end{aligned}
$$

Writing $A$ as $\left[\begin{array}{ll}a & b \\ c & d\end{array}\right],(19)$ gives 8 equations, which break up naturally into 2 sets:

$$
\begin{aligned}
& a \alpha_{-1}+b \gamma_{-1}=0 \\
& a \beta_{-1}+b \delta_{-1}=0 \\
& \text { (I) } \\
& a \alpha_{0}+b \gamma_{0}=-\alpha_{-1} \\
& c \alpha_{-1}+d \gamma_{-1}=0 \\
& a \beta_{0}+b \delta_{0}=-\beta_{-1} \\
& c \alpha_{0}+d \gamma_{0}=-\gamma_{-1} \\
& a \beta_{-1}+b \delta_{-1}=0 \\
& a \beta_{0}+b \delta_{0}=-\delta_{-1}
\end{aligned}
$$

If $\chi$ is invertible, then the first version of $A$ given in (18) above can be formed, and it is easily checked that the $a, \ldots, d$ so determined satisfy (I) above. We must check that (II) is satisfied as well. Suppose $\alpha_{-1}$ and $\delta_{-1}$ are also invertible. Then (20.3) shows that $\gamma_{-1}$ and $\beta_{-1}$ are invertible, and (20.1) gives

$$
\delta_{-1}=\alpha_{-1}^{t^{-1}} \gamma_{-1}^{t} \beta_{-1}=\gamma_{-1} \alpha_{-1}^{-1} \beta_{-1}, \quad \alpha_{-1}=\beta_{-1} \delta_{-1}^{-1} \gamma_{-1} .
$$

We now claim that

$$
\chi \alpha_{-1}^{-1} \beta_{-1}=\gamma_{-1}^{t} \delta_{-1}^{t^{-1}} \hat{\chi}
$$

Writing out the left-hand side of (20.5), we get

$$
\left[\alpha_{0}^{t} \gamma_{-1}-\gamma_{0}^{t} \alpha_{-1}\right] \alpha_{-1}^{-1} \beta_{-1}=\alpha_{0}^{t} \gamma_{-1} \alpha_{-1}^{-1} \beta_{-1}-\gamma_{0}^{t} \beta_{-1}=\alpha_{0}^{t} \delta_{-1}-\gamma_{0}^{t} \beta_{-1},
$$

where we have used (20.4) and $\chi=\chi^{t}$. Similarly, the right-hand side of (20.5) gives $\gamma_{0}^{t} \beta_{-1}-\alpha_{0}^{t} \delta_{-1}$, and the two expressions are identical by virtue of (20.3). This shows that $\hat{\chi}$ is invertible and allows us to write down the second expression for $A$, which involves $\hat{\chi}^{-1}$. It is then easily checked, using (20.4) and (20.5), that the two expressions are identical and the rest of the proof follows.

Setting $G_{-}(\lambda):=I-(\lambda-p)^{-1} A$, and $G_{+}^{-1}(\lambda):=\left[I+(\lambda-p)^{-1} A\right] \cdot G(\lambda)$ (recall that $A^{2}=0$ ), we have solved the Riemann-Hilbert problem for $G(\lambda)$ on any positively oriented contour in $D \backslash\{p\}$ equivalent to $\partial D$. Moreover, $G_{-}(\lambda)$ is the unique solution with $G_{-}(\infty)=I$. (See Novikov, et. al. [NMPZ] for a general discussion.) Finally, we observe that both of $G_{ \pm}(\lambda)$ take values in $\operatorname{Sp}(k, \mathbb{C})$ in their respective domains. 


\section{The Riemann-Hilbert Problem for Self-Dual Yang-Mills Fields}

Returning now to $G(x, \lambda)$, choose a simple, positively oriented contour $\mathscr{C}_{x}$ on $L_{x}$ surrounding the $n$ points of $V_{a} \cap L_{x}$. Order these points as $\left\{p_{1}(x), p_{2}(x), \ldots, p_{n}(x)\right\}$. Choose contours $\mathscr{C}_{i}$ to surround only $\left\{p_{1}(x), p_{2}(x), \ldots, p_{i}(x)\right\}$, with $\mathscr{C}_{n}=\mathscr{C}_{x}$. Let $D_{i}$ be the closure of int $\mathscr{C}_{i}$. The following construction works for a generic $x$ (see the remarks below): On $D_{1} \backslash\left\{p_{1}(x)\right\}$ apply the lemma to get

$$
G(x, \lambda)=\left(I-\left(\lambda-p_{1}(x)\right)^{-1} A_{1}(x)\right) \cdot G_{1}(x, \lambda),
$$

with $G_{1}(x, \lambda)$ holomorphic in $D_{1}$, hence in $D_{2} \backslash\left\{p_{2}(x)\right\}$, and taking values in $\operatorname{Sp}(k, \mathbb{C})$. Continue with $G_{i-1}(x, \lambda)$ in $D_{i} \backslash\left\{p_{i}(x)\right\}$ to obtain

$$
G_{i-1}(x, \lambda)=\left(I-\left(\lambda-p_{i}(x)\right)^{-1} A_{i}(x)\right) \cdot G_{i}(x, \lambda)
$$

or, equivalently,

$$
G(x, \lambda)=\left(\prod_{j=1}^{i}\left[I-\left(\lambda-p_{j}(x)\right)^{-1} A_{j}(x)\right]\right) \cdot G_{i}(x, \lambda)
$$

arriving at the factorization

$$
G(x, \lambda)=\left(\prod_{j=1}^{n}\left[I-\left(\lambda-p_{j}(x)\right)^{-1} A_{j}(x)\right]\right) \cdot G_{n}(x, \lambda)=G_{-}(x, \lambda) \cdot G_{+}^{-1}(x, \lambda)
$$

valid on the original curve $\mathscr{C}_{x}$.

Remarks. At each stage of the factorization, one must restrict the domain of $x$ further by cutting out the Zariski-closed subsets of $\operatorname{Gr}(2,4)$ in which the $k \times k$ determinants of the required terms made from the Laurent expansion of $G$ vanish. The end result of this process is the set $\mathscr{U}$ referred to above. Evidently, $G_{-}$is holomorphic in $\hat{\mathfrak{U}}_{a} \mid \mathscr{U}$. Since the $A_{i}$ are nilpotents of order $2, G_{-}^{-1}=\prod_{j=n}^{1}(I+$ $\left.\left(\lambda-p_{j}(x)\right)^{-1} A_{j}(x)\right)$, so that $G_{-}^{-1} \cdot G=G_{+}^{-1}$ is holomorphic in $\hat{\mathfrak{U}}_{b} \mid \mathscr{U}$, and the problem is solved.

Even for the case $k=1(\operatorname{Sl}(2, \mathbb{C}))$, the stated conditions are not necessary, only sufficient. This can be seen from the following example for $n=2, k=1$. Take

$$
\mathscr{A}^{t}(Z)=\left[\begin{array}{cccccc}
Z_{0} & Z_{2} & Z_{1} & Z_{3} & 0 & 0 \\
Z_{2} & Z_{0} & Z_{3} & Z_{1} & Z_{2} & \varepsilon Z_{3}
\end{array}\right] \text {, with } \varepsilon \neq 0 .
$$

Using (12), we find

$$
G_{12}(Z)=\left[\begin{array}{cc}
0 & -1 \\
1 & 0
\end{array}\right]+\frac{Z_{0} Z_{1}+Z_{2} Z_{3}}{\left(Z_{1}^{2}-Z_{3}^{2}\right)\left(Z_{0}^{2}-Z_{2}^{2}\right)}\left[\begin{array}{cc}
Z_{2}^{2} & \varepsilon Z_{2} Z_{3} \\
\varepsilon Z_{2} Z_{3} & \varepsilon^{2} Z_{3}^{2}
\end{array}\right] .
$$

Using the standard coordinates $x=\left[\begin{array}{cc}y & -\tilde{z} \\ z & \tilde{y}\end{array}\right]$, one finds, after a routine computation at the pole $p_{1}(x)=z /(1-\tilde{y})$, that

$$
\chi\left(p_{1}(x)\right)=\frac{2(\varepsilon-1)}{1+z \tilde{z}+y \tilde{y}-(y+\tilde{y})}
$$


which evidently vanishes when $\varepsilon=1$. On the other hand, for $\varepsilon=1$, we find immediately that

$$
G_{12}(Z) \mid L_{0}=\left[\begin{array}{cc}
\lambda^{-1} & 0 \\
2 & \lambda
\end{array}\right] \sim I_{2}
$$

so that $E$ is, in fact, trivial over the generic line. We mention that if $\varepsilon \neq 1$, there are no difficulties, and the construction goes through as advertised.

The restriction to "generic $G$ " in the statement of the theorem eliminates the possibility that one or more of the determinants may vanish identically and ensures that $\Delta_{a}$ and $\Delta_{b}$ are relatively prime.

In this procedure, one introduces "artificial" singularities into the gauge potential; this happens as well in the Atiyah-Ward construction [At]. The Riemann-Hilbert problem may be solvable for some of these $x$ with a different ordering of the singular points, or, since we only have a sufficient condition, it might be solvable in a different form. In addition, requiring that $L_{x}$ be in general position with respect to the pair $\left(V_{a}, V_{b}\right)$ cuts out another set of the Grassmannian on part of which the problem might be solvable.

Although $G_{-}(x, \lambda)$ is certainly unique, the factorization is not; it depends on the (arbitrary) ordering of the singular points. Indeed, for certain values of $x$, the factorization exists for one such ordering and fails for another.

\section{Backlund Transformations Associated with the Factorization}

At this point, we may choose to forget that we know where to put the contour $\mathscr{C}_{x}$, and observe that for each $i$, the partial factorization given in (23) above permits the construction of a sequence of self-dual gauge potentials $\Gamma_{i A B} d x^{A B}$ via

$$
\Gamma_{i A}(x, \pi)=G_{i}^{-1}(x, \pi) \cdot D_{A} G_{i}(x, \pi)
$$

with

$$
G_{i}(x, \pi)=\prod_{j=1}^{i}\left(I-\left(\lambda-p_{j}(x)\right)^{-1} A_{j}(x)\right)
$$

obtained by solving the Riemann-Hilbert problem on the contour $\mathscr{C}_{i}$. The potentials are related by the Bäcklund transformations (or "dressing transformations" of Zakharov \& Shabat) [ZS, Ch, PSW, Cr, MCN]:

$$
\Gamma_{i A}=G_{i}^{-1} \cdot \Gamma_{i-1 A} \cdot G_{i}+G_{i}^{-1} D_{A} G_{i} .
$$

For $i<n$, these hold for $x$ in supersets of $\mathscr{U}$. The potential $\Gamma_{i}$ may be regarded as having been obtained by $i$ successive such transformations applied to the trivial solution $\Gamma_{0}=I$. Of course, these transformations are not well-defined on isomorphism classes of bundles; they do not, for example, respect topological invariants like Chern classes.

To relate this to the standard treatments of the Riemann problem [NMPZ], set $\phi_{j}=I-\left(\lambda-p_{j}(x)\right)^{-1} A_{j}(x)$ and consider the situation at the $i^{\text {th }}$ stage of the induction. On the contour $\mathscr{C}_{i}$, we need to solve the problem $G_{i-1}=\phi_{i} \cdot G_{i}$; equivalently, we have the singular solution $G=\phi_{1} \phi_{2} \cdots \cdot \phi_{i-1} \cdot G_{i-1}(x, \lambda)$ to the "Riemann problem with zeros" for $G$ on $\mathscr{C}_{i}$. If a regular solution exists, it differs 
from this by the interpolation of a factorization of $I$; this is exactly what we get, since

$$
G=\left(\phi_{1} \cdots \cdot \phi_{i-1} \cdot \phi_{i}\right) \cdot\left(\phi_{i}^{-1} \cdot G_{i-1}\right) .
$$

As mentioned earlier, an analogous result holds for the even dimensional orthogonal groups $O(2 k, \mathbb{C})$. Here one takes the quadratic form

$$
Q=\left[\begin{array}{cc}
Q_{n} & 0 \\
0 & Q_{k}
\end{array}\right], \text { where } Q_{m}=\left[\begin{array}{cc}
0 & I_{m} \\
I_{m} & 0
\end{array}\right]
$$

on $\mathbb{K}$ and requires that $\operatorname{Im} \mathscr{A}(Z)$ be totally null with respect to $Q$. Everything goes through with the obvious modifications.

As was also mentioned, the factorization does not depend on $G_{a b}(Z)$ having come from the monad construction. It is only necessary that $G$ have the correct form (meromorphic with a finite number of simple poles that can be isolated from the rest on some open subset of the Grassmannian). These factorizations are quite similar to those obtained recently [Uh] for harmonic maps.

Finally, we mention a simple relation between the nilpotents $A_{i}(x)$ and the gauge potential $\Gamma$. In the standard coordinates $x=\left[\begin{array}{rr}y & -\tilde{z} \\ z & \tilde{y}\end{array}\right]$, the normalization $G_{-}(x, \infty)=I$ gives $\Gamma_{y}(x)=\Gamma_{z}(x)=0$. Sufficiently far from $\lambda=0$, we can, for each $x$ and $i$, write $\left[\lambda-p_{i}(x)\right]^{-1}=\lambda^{-1}+\lambda^{-2} p_{i}(x)+O\left(\lambda^{-2}\right)$, so that

Now (15) gives

$$
G_{-}(x, \lambda)=I+\lambda^{-1}\left(\sum_{1}^{n} A_{i}(x)\right)+\lambda^{-2} H\left(x, \lambda^{-1}\right) \text {. }
$$

$$
\begin{aligned}
& \left(\partial_{y}+\lambda^{-1} \partial_{\tilde{z}}\right) G_{-}=\lambda^{-1} G_{-} \cdot \Gamma_{\tilde{z}}, \\
& \left(\partial_{z}-\lambda^{-1} \partial_{\tilde{y}}\right) G_{-}=-\lambda^{-1} G_{-} \cdot \Gamma_{\tilde{y}},
\end{aligned}
$$

and, equating the lowest order coefficients we get

$$
\partial_{y}\left(\sum_{1}^{n} A_{i}(x)\right)=\Gamma_{\tilde{z}}, \quad \text { and } \quad \partial_{z}\left(\sum_{1}^{n} A_{i}(x)\right)=-\Gamma_{\tilde{y}}
$$

as the infinitesimal version of the factorization. The quantity $Q_{1}(x)=\left(\sum_{1}^{n} A_{i}(x)\right)$ is the first of an infinite number of conserved "charges" for the self-dual Yang-Mills fields $[\mathrm{Ch}, \mathrm{Ta}]$.

\section{References}

[ADHM] Atiyah, M. F., Drinfeld, V. G., Hitchin, N. J., Manin, Yu. I.: Phys. Lett. 65A, 185-187 (1978)

[At] Atiyah, M. F.: Geometry of Yang-Mills fields. Fermi Lectures (1978)

[AW] Atiyah, M. F., Ward, R. S.: Commun. Math. Phys. 55, 117-124 (1977)

[Ch] Chau, L. L.: Geometric integrability and equations of motion in physics. In: Integrable Systems. Song Xing-Chang (ed.). Singapore: World Scientific 1988

[Cr] Crane, L.: Commun. Math. Phys. 110, 391-414 (1987)

[Do] Donaldson, S.: Commun. Math. Phys. 93, 453-460 (1984)

[CFGY] Corrigan, E., Fairlee, D. B., Goddard, P., Yates, R. G.: Commun. Math. Phys. 58, 223 (1978) 
[MCN] Mason, L., Chakravarty, S., Newman, E. T.: J. Math. Phys. 29, 1005-1013 (1988)

[NMPZ] Novikov, S., Manakov, S. V., Pitaevski, L. P., Zakharov, V. E.: Theory of solitons. New York: Plenum Press 1984

[OSS] Okonek, C., Schneider, M., Spindler, H.: Vector bundles on complex projective spaces. Boston: Birkhäuser 1980

[PR] Penrose, R., Rindler, W.: Spinors and space-time, vol. 2. Cambridge: Cambridge University Press 1986

[PSW] Prasad, M. K., Sinha, A., Wang, L. L.: Phys. Lett. B87, 237 (1979)

[Ta] Takasaki, K.: Commun. Math. Phys. 94, 35-59 (1984)

[Uh] Uhlenbeck, K.: J. Diff. Geom. 30, 1-50 (1989)

[Wa] Ward, R. S.: Phys. Lett. 61A, 81-82 (1977)

[We] Wells, R. O. Jr.: Bull. AMS (new series) 1, 296-336 (1979)

[ZS] Zakharov, V. E., Shabat, A. B.: Funkts. Analiz. 13(3), 13-22 (1978)

Communicated by S.-T. Yau

Received November 2, 1989 
